\title{
Clinicopathologic Correlation of Oral Lichen Planus and Oral Lichenoid Lesions: A Preliminary Study
}

\author{
Marinka Mravak-Stipetić, ${ }^{1}$ Božana Lončar-Brzak, ${ }^{1}$ Iva Bakale-Hodak, ${ }^{1}$ Ivan Sabol, ${ }^{2}$ \\ Sven Seiwerth, ${ }^{3}$ Martina Majstorović, ${ }^{4}$ and Magdalena Grce ${ }^{2}$ \\ ${ }^{1}$ Department of Oral Medicine, Department of Oral Medicine, School of Dental Medicine, University of Zagreb, 10000 Zagreb, Croatia \\ ${ }^{2}$ Division of Molecular Medicine, Rudjer Boskovic Institute, 10000 Zagreb, Croatia \\ ${ }^{3}$ Department of Pediatric Dentistry, School of Dental Medicine, University of Zagreb, 10000 Zagreb, Croatia \\ ${ }^{4}$ School of Medicine, Institute of Pathology, University of Zagreb, 10000 Zagreb, Croatia
}

Correspondence should be addressed to Marinka Mravak-Stipetić; mmstipetic@gmail.com

Received 31 July 2014; Revised 8 September 2014; Accepted 17 September 2014; Published 29 October 2014

Academic Editor: Niroshani Soysa

Copyright (c) 2014 Marinka Mravak-Stipetić et al. This is an open access article distributed under the Creative Commons Attribution License, which permits unrestricted use, distribution, and reproduction in any medium, provided the original work is properly cited.

\begin{abstract}
Oral lichen planus (OLP) and oral lichenoid lesions (OLL) are clinically and histologically similar lesions but their treatment planning and prognosis are different. The review of the literature indicates numerous criteria to distinguish these two lesions; however there is a lot of inconsistency. Thus, the aim of this study was to determine the correlation of histopathology and clinical OLP and OLL diagnosis and to clarify which histopathologic criteria could best distinguish these two diagnoses. A retrospective study showed that clinically diagnosed 92 OLPs and 14 OLLs have been confirmed histopathologically in $52.2 \%$ and $42.9 \%$ of cases, respectively. In addition, histopathology showed statistically significant more eosinophils $(P<0.0005)$, plasma cells $(P<0.0005)$, and granulocytes $(P<0.05)$ in OLL than OLP. To establish histopathological diagnosis of OLP and OLL it should be mandatory to define the type of cells in mononuclear infiltrate, which can be associated more accurately with clinical feature and patient history. Therefore, currently accepted diagnostic criteria for OLP and OLL should be modified and validated on a larger number of patients taking into account particular distinguishing histopathological features.
\end{abstract}

\section{Introduction}

Oral lichen planus (OLP) is a chronic immunological mucocutaneous disorder of unknown etiology with prevalence in general population ranging from 0.2 to $2 \%[1,2]$, whereas the prevalence of OLP found in our population was $4.3 \%$ [3]. Due to low $[2,4,5]$ and rather questionable [6-8] risk for malignant transformation, OLP is classified as potentially malignant disorder [9]. Indeed, OLP has distinctive clinical features within the oral cavity with several characteristic morphological types and symmetrical distribution in oral mucosa $[5,6,8]$. These features facilitate clinical diagnosis. However, due to its premalignant potential, nonreticular types, unilateral presentation, or lesions present at the cancerrisk oral sites it requires histopathological confirmation as well.
Histopathological criteria for OLP, which are currently accepted in clinical practice, are those given by WHO [10]. These criteria do not distinguish between the OLP and oral lichenoid lesions (OLL); therefore, several reports have suggested their modifications $[8,11,12]$. Van der Meij and van der Waal [11] proposed modified WHO criteria according to which OLP could be diagnosed only in cases when both clinical and histopathological criteria are fulfilled, while in other cases, the disorder should be considered as OLL.

It is accepted that OLL is clinically and histologically similar to OLP [11,13] but has less characteristic morphology and distribution, whereas lichen planus-like lesions are described as oral lichenoid lesion (OLL), oral lichenoid tissue reaction (OLTR), or lichenoid contact stomatitis [14]. The etiology of OLL is usually identifiable: in case of topographically related lesions to amalgam fillings, intake of particular drugs, history 
of chronic graft versus host disease (cGvHD), or systemic diseases $[4,15]$. OLL is also considered as potentially malignant disorder [4], although it is still unclear whether its malignant potential differs from OLP [16]. So far, various authors [1, $2,14,17]$ have suggested different parameters that might be indicative for OLP and OLL diagnosis (Table 1). Given that the approach to treatment planning and prognosis of both OLP and OLL usually differs, the suggested parameters define criteria that distinguish these lesions.

As opposed to rather evident clinical diagnosis of OLP, less obvious, imprecisely or incompletely defined histopathological findings often complicate the confirmation of final diagnosis. Therefore, the aim of this preliminary study was to determine the correlation of histopathological and clinical diagnosis of OLP and OLL and to clarify which histopathologic features distinguish best these two diagnoses and could improve differential histopathological diagnosis.

\section{Materials and Methods}

The retrospective study group comprised 106 patients who were referred to the Department of Oral Medicine, School of Dental Medicine, University of Zagreb, in the period between January 1, 2012, and December 31, 2012. Diagnosis was based on clinical examination and medical and dental history, intake of drugs, and duration of the lesions. Clinical diagnoses were established by experienced (MMS) and trained clinicians (BLB, IB), specialist in oral medicine who used the same and consistent criteria in scoring levels of OLP and OLL. Histopathologic diagnosis was established at a single pathology service that is under supervision of an experienced pathologist (SS). In all patients with OLP and OLL oral mucosa swabs for yeast culture on Sabouraud Agar plates (Sabouraud Dextrose Agar (Becton Dickinson and Co., Cockeysville, USA) were taken, as a routine procedure in all patients with white oral lesions. Those who have had positive finding of yeast superinfection were excluded from the study. Only patients who underwent biopsy were included in the study. The clinical diagnosis of OLP was established in 92 patients (70 female; 22 male; ratio $\mathrm{f} / \mathrm{m}=3.18: 1$ ), while in 14 patients OLL was diagnosed ( 6 female; 8 male, ratio $\mathrm{f} / \mathrm{m}=$ $0.75: 1)$. The mean age of OLP and OLL patients was 56.1 and 64.9 years, respectively.

OLP was diagnosed according to criteria described by Kramer et al. [10]: presence of white papules and/or striae usually with bilateral involvement and histopathological signs of liquefaction degeneration in the basal cell layer (degenerative changes to the basal cells) along with the presence of a well-defined band-like zone of inflammatory infiltrate confined to the superficial part of the connective tissue (this infiltrate being composed almost exclusively of lymphocytes and characterized by absence of epithelial dysplasia). Clinical diagnosis of OLL was established due to the presence of hyperkeratotic lesions adjacent to amalgam fillings with asymmetric and mainly unilateral distribution and a patient medical history related to drugs, which provoke lichenoid changes in oral mucosa [16].

Data were analyzed by using Chi-squared test $\left(\chi^{2}\right)$ and differences at $P<0.05$ were considered to be significant.

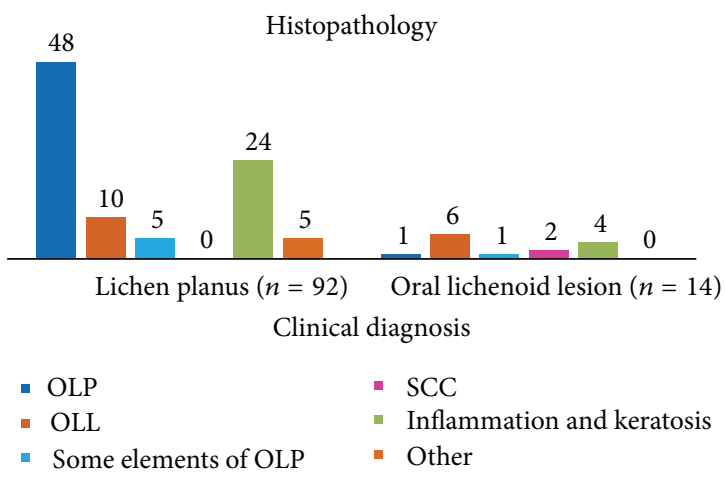

FIGURE 1: The distribution of clinical and histopathologic diagnoses among patients; OLP: oral lichen planus; OLL: oral lichenoid lesions; OSCC: oral squamous cell carcinoma.

\section{Results}

The distribution of clinical and histopathological diagnoses is shown on Figure 1. In 52.2\% (48/92) of patients, clinical diagnosis of OLP was histopathologically confirmed, while in $5.4 \%$ (5/92) of cases there was a partial confirmation and only some criteria were fulfilled. In $10.9 \%$ (10/92) of OLP patients, both clinical and histopathologic diagnosis were concordant, while in $26.1 \%$ (24/92) of patients histopathologic diagnosis was nonspecific, being described as inflammation and keratosis. Clinical and histopathological diagnoses coincide in $42.9 \%(6 / 14)$ of OLL patients. According to the histopathologic findings, in clinically diagnosed OLL, in one case the diagnosis of OLP was established, one have had some histopathological elements of OLP, and 4 (28.6\%) cases had inflammation and keratosis, while in $2(14.3 \%)$ cases squamous cell carcinoma (OSCC) was diagnosed.

The distribution of histopathologic features between OLP and OLL with statistically significant differences between the parameters is indicated in Figure 2. Results showed significantly more eosinophils $(P<0.0005)$, plasma cells $(P<0.0005)$, and granulocytes $(P<0.05)$ in OLL than OLP. Other observed parameters such as premature keratosis and inflammatory cell invasion through basal membrane were more frequently found in OLP and OLL in comparison with nonspecific clinical findings of inflammation and keratosis; the difference was significant $(P<0.005$ and $P<0.0005$, resp.). Abundant and diffuse mononuclear infiltration was more frequently found in cases of inflammation and keratosis than in OLP or OLL with statistically significant differences $(P<0.05, P<0.005$, or $P<0.0005)$, but between OLP and OLL diagnosis statistically significant difference in these parameters was not found.

\section{Discussion}

To establish and confirm OLP and OLL diagnosis by using methods such as clinical examination and histopathological analysis, which are available in everyday clinical practice and among wider population of patients, sometimes represents a diagnostic challenge. Earlier reports have shown that while 
TABLE 1: Histopathological criteria distinctive of OLP and OLL.

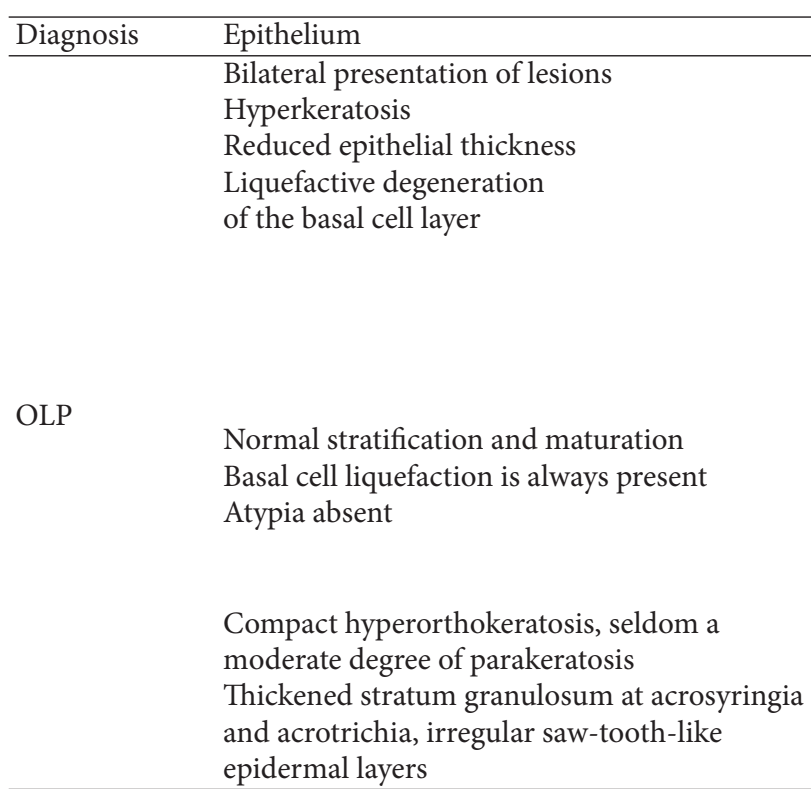

Unilateral presentation of lesions

OLL

Focal parakeratosis, cytoid bodies in the cornified layer

Thickened stratum granulosum possible, however, with focal interruption of the granular layer, cytoid bodies in the granular layer, necrotic keratinocytes scattered in all epidermal layers

OLL related with amalgam filling

Normal stratification basal cell liquefaction may or may not be present Atypia absent

Basal cell liquefaction may not be present

$\begin{array}{lc}\text { Subepithelium } & \text { Authors } \\ \begin{array}{l}\text { Well-defined subepithelial band of chronic } \\ \text { inflammatory infiltrate composed predominantly of }\end{array} & \begin{array}{l}\text { Juneja et al., } \\ \text { lymphocytes }\end{array} \\ 2006 \text { [14] }\end{array}$

Absence of eosinophils and neutrophils

Band shaped inflammatory infiltrate in some or all areas

Thornhill et al. 2006 [1]

Dense band of inflammatory components, chiefly

lymphocytes, in the juxtaepithelium

Dyskeratotic epithelial cells

Ismail et al.

Civatte bodies are usually found in subepithelial area, at

2007 [22]

the junction of the lamina propria and epithelial layer

Mostly a superficial dermal inflammatory infiltrate;

seldom eosinophils

Ziemer, 2014

acanthosis, necrotic keratinocytes in the lower epidermal layers
Poorly differentiated lower border of the subepithelial inflammatory zone

Presence of a substantial number of plasma cells in the lymphocytic infiltrate

Perivascular infiltrate

Juneja et al.,

Increased number of colloid bodies

Presence of acute inflammatory cells, such as

eosinophils and neutrophils

More often a deep dermal infiltrate, especially in nonphotodistributed lichenoid drug eruption; admixture of eosinophils and plasma cells possible (presence of plasma cells is a regular finding in biopsies from mucous membranes independently of the origin of dermatosis)

\section{Inflammatory infiltrate located deep to superficial} infiltrate in some or all areas

Focal perivascular infiltrate

Plasma cells, eosinophils, and neutrophils in the

Thornhill et al., 2006 [1] connective tissue

Lymphoid follicle formations, with mixed inflammatory

Ismail et al.

cells consisting of plasma cells and neutrophils

2007 [22]

Predominant formation of lymphoid follicles chiefly consisting of plasma cells and neutrophils Dense inflammatory cells in the stroma

Hiremath et al., $2011[2]$

Extensive degeneration in the lower prickle cell layer, prompting spongiotic vesicle formation Basal cell liquefaction is usually present Atypia absent

Infiltrate is not band-like but extends to the deeper stroma inflammatory cells predominated by plasma cells and eosinophils

Ismail et al.

Perivascular cuffing of inflammatory cells is evident 2007 [22]

Infiltrate is often not band-like but extends to the deeper stroma, with plasma cells and eosinophils which predominate the inflammatory component
Hiremath et al., $2011[2]$ 


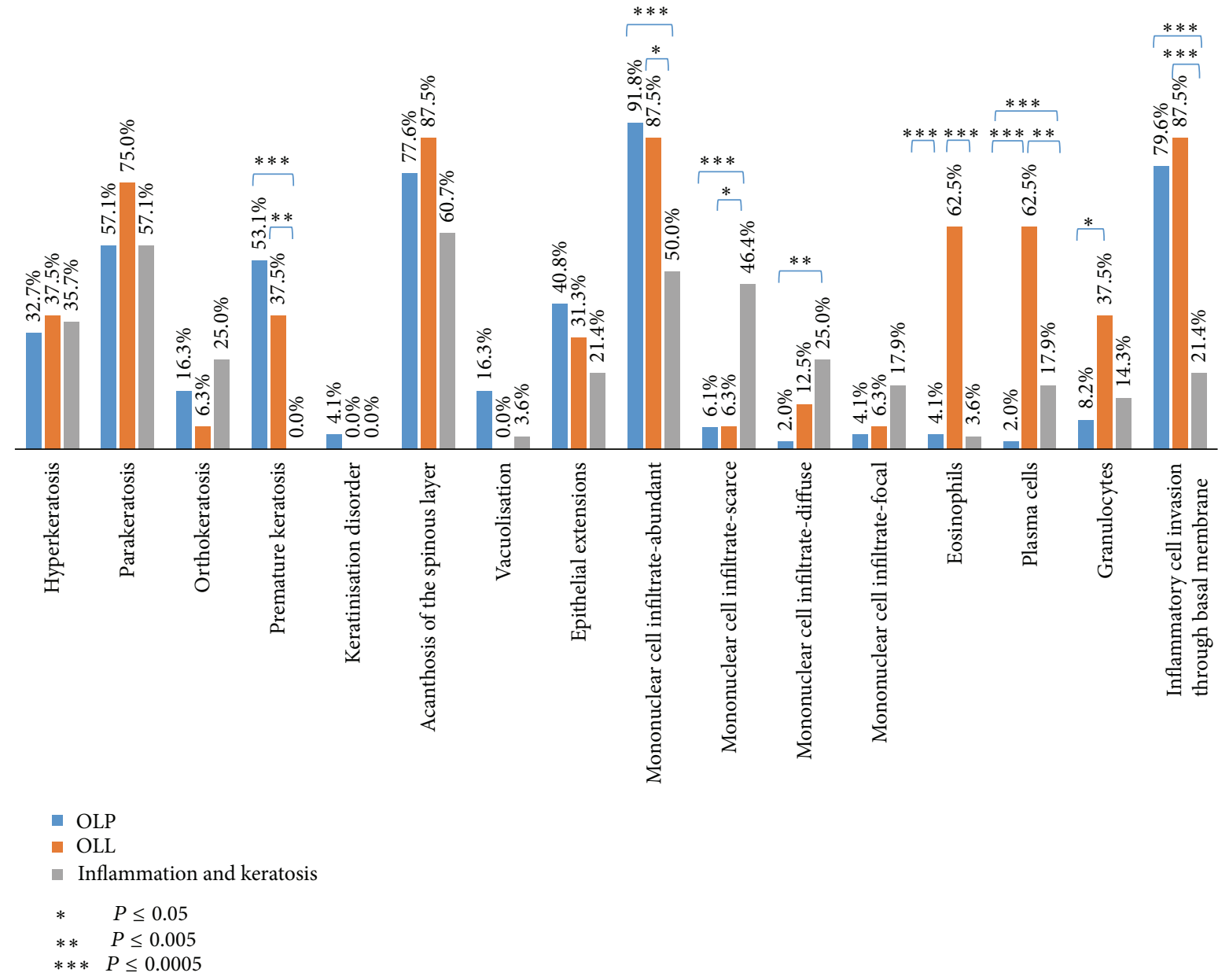

Figure 2: The distribution of histopathologic criteria between the diagnosis oral lichen planus (OLP) and oral lichenoid lesion (OLL).

clinical diagnosis depends on a clinician interpretation [11, 14], histopathological diagnosis is strictly dependent on a pathologist interpretation as well $[2,12]$, but also the choice of biopsy area [18], clinical severity of the disease, activity or remission of the disease, and the clinical type of OLP (reticular lesions are considered easier for histopathological confirmation) [2, 19]. Pathologists' lack of information on clinical features and distribution of lesions could also influence their judgment $[11,12,20]$. Having in mind these parameters, which could affect the final histopathologic interpretation, the results of our study could partially be explained by possible interobserver bias as the patients were examined by different clinicians, and histopathological diagnosis was done by different pathologists. This should be taken into account in the future prospective studies. Therefore, due to many variables affecting diagnosis, histopathological finding is insufficiently reproducible $[2,12]$.

The results of this study show that clinical diagnoses of OLP and OLL have been confirmed histopathologically in $52.2 \%$ patients with OLP and $42.9 \%$ patients with OLL. These results are similar to those shown by van der Meij and van der Waal [11] according to whom $42 \%$ of OLP clinically diagnosed cases were not confirmed by histopathology.
Interestingly, authors also showed that a clinician's consensus in patients with clear histopathological confirmation of OLP was achieved in only $50 \%$ of cases. Thornhill et al. [1] showed an overall correlation of clinical and histopathological diagnoses of OLP and OLL based on the findings of five different pathologists. Difficulties reported in distinguishing these lesions in histological features were related to amalgam fillings in only $36 \%$ of cases. These results are similar to those of Al-ani [20] who found clinical and pathological correlation of OLP in only $38.5 \%$. Rad et al. [21] found significantly higher clinical and pathological correlation of OLP (93.9\%) in cases in which WHO modified criteria were applied. The results of Rad et al. [21] are promising but should be validated in a larger group of patients, applying strictly validated and reproducible criteria [11].

According to the literature review, there is a lack of studies, which applied WHO modified criteria in determining correlation of clinical and histopathological diagnosis of OLP. It would be reasonable to expect that the overall prevalence of OLP in the observed population would be lower and the prevalence of OLL higher if WHO modified criteria for establishing diagnosis were applied. This could possibly affect an expected malignant transformation rate in both 
OLP and OLL showing that OLL is more prone to malignant transformation than OLP as already documented by some authors $[8,22]$ and also shown in our results.

Van der Meij et al. [23, 24] have found in prolonged follow-up that patients with OLL have an increased risk for malignant transformation compared to OLP and that this is more likely to occur in erosive lesions. The malignant transformation could be attributable to the extrinsic factors [24], other than alcohol and tobacco, use of particular antihypertensive [25] and antiretroviral drugs [26], and infection with strains of Candida albicans [27], which all can enhance malignant transformation in oral lesions. In our two patients with oral SCC we did not found any of the listed risk factors. This finding emphasises the importance of regular followup of patients with oral lesions and eventually biopsies to confirm or refute clinical diagnosis of OLL or OLP.

Gannot et al. [28] have shown that tissue changes towards malignancy had a distinct lymphocyte profile: the number of CD4, CD8, and B cells is significantly higher in inflammatory infiltrate in moderate and severe dysplasia and SCC, compared to mild dysplasia and hyperkeratosis. In addition, inflammatory cell infiltration has been considered in cancer progression. Particularly, CD8 and NK cell are increased in oral SCC since they have cytotoxic immune response against neoplastic cells [29].

Several authors so far have suggested histopathologic criteria, which should be distinctive in achieving both OLP and OLL diagnoses (Table 1). In their studies, they found differences in some parameters between OLP and OLL, but in order to accept these findings as a standard protocol, a larger number of patients should be examined as already suggested by some authors. All authors agree that findings of plasma cells, eosinophils, and neutrophils in inflammatory infiltrate can be distinctive for OLL compared to OLP, which is in consensus with our results (Figure 2). Therefore, we consider that this type of cells should be a certain diagnostic feature in histopathologic differentiation between OLP and OLL given that these cells are relevant in nonimmediate allergic reactions, particularly those induced by drugs [30,31], where OLL is classified.

Unlike other findings (Table 1), we did not observed statistically significant differences in other histopathological criteria between OLP and OLL. These results therefore may enhance a closer collaboration with pathologists in order to get a more precise description of histopathological features, particularly cellular composition of mononuclear infiltrate. This consequently should improve the list of parameters, which pathologists may consider relevant for either diagnosis, therefore avoiding misinterpretation due to different criteria, which usually affect the results.

\section{Conclusion}

OLP and OLL are clinically similar but diagnostically insufficiently distinguished lesions. Their prognosis and treatment may vary. To achieve accurate histopathologic diagnosis it is mandatory to define the type of cells in mononuclear infiltrate, which can be interpreted in accordance with clinical findings and a patient history. Currently accepted diagnostic criteria in diagnosing OLP and OLL should be modified and modifications should be validated in a larger number of patients. The results of this study, which is in accordance with similar reports from the literature, have shown that histopathological findings coincide with clinical diagnosis in approximately only $50 \%$ of cases and as such cannot always be exclusive in the final interpretation. Based on the results of this study, prominent diagnostic histopathological features in distinguishing between OLP and OLL are the type of cells in the mononuclear cell infiltrate, that is, eosinophils, plasma cells, and granulocytes.

\section{Conflict of Interests}

The authors declare that there is no conflict of interests regarding the publication of this paper.

\section{Acknowledgment}

This research has been supported by the National Science Foundation of Croatia.

\section{References}

[1] M. H. Thornhill, V. Sankar, X.-J. Xu et al., "The role of histopathological characteristics in distinguishing amalgamassociated oral lichenoid reactions and oral lichen planus," Journal of Oral Pathology and Medicine, vol. 35, no. 4, pp. 233240, 2006.

[2] S. K. S. Hiremath, A. D. Kale, and S. Charantimath, "Oral lichenoid lesions: clinico-pathological mimicry and its diagnostic implications," Indian Journal of Dental Research, vol. 22, no. 6, pp. 827-834, 2011.

[3] B. L. Brzak, M. Mravak-Stipetić, I. Canjuga et al., "The frequency and malignant transformation rate of oral lichen planus and leukoplakia-a retrospective study," Collegium Antropologicum, vol. 36, no. 3, pp. 773-777, 2012.

[4] I. van der Waal, "Oral lichen planus and oral lichenoid lesions; a critical appraisal with emphasis on the diagnostic aspects," Medicina Oral, Patologia Oral y Cirugia Bucal, vol. 14, no. 7, pp. E310-E314, 2009.

[5] C. Scully and M. Carrozzo, "Oral mucosal disease: Lichen planus," British Journal of Oral and Maxillofacial Surgery, vol. 46, no. 1, pp. 15-21, 2008.

[6] F. A. C. G. De Sousa and L. E. B. Rosa, "Oral lichen planus: clinical and histopathological considerations," Brazilian Journal of Otorhinolaryngology, vol. 74, no. 2, pp. 284-292, 2008.

[7] D. J. Krutchkoff, L. Cutler, and S. Laskowski, "Oral lichen planus. The evidence regarding potential malignant transformation," Journal of Oral Pathology, vol. 7, no. 1, pp. 1-7, 1978.

[8] E. Eisenberg, "Oral lichen planus: a benign lesion," Journal of Oral and Maxillofacial Surgery, vol. 58, no. 11, pp. 1278-1285, 2000.

[9] I. van der Waal, "Potentially malignant disorders of the oral and oropharyngeal mucosa; terminology, classification and present concepts of management," Oral Oncology, vol. 45, no. 4-5, pp. 317-323, 2009.

[10] I. R. H. Kramer, R. B. Lucas, J. J. Pindborg, and L. H. Sobin, "Definition of leukoplakia and related lesions: an aid to 
studies on oral precancer," Oral Surgery Oral Medicine and Oral Pathology, vol. 46, no. 4, pp. 518-539, 1978.

[11] E. H. van der Meij and I. van der Waal, "Lack of clinicopathologic correlation in the diagnosis of oral lichen planus based on the presently available diagnostic criteria and suggestions for modifications," Journal of Oral Pathology and Medicine, vol. 32, no. 9, pp. 507-512, 2003.

[12] S.-O. Piboonniyom, N. Treister, W. Pitiphat, and S.-B. Woo, "Scoring system for monitoring oral lichenoid lesions: a preliminary study," Oral Surgery, Oral Medicine, Oral Pathology, Oral Radiology and Endodontology, vol. 99, no. 6, pp. 696-703, 2005.

[13] D. Eisen, M. Carrozzo, J.-V. B. Sebastian, and K. Thongprasom, "Number V. Oral lichen planus: clinical features and management," Oral Diseases, vol. 11, no. 6, pp. 338-349, 2005.

[14] M. Juneja, S. Mahajan, N. N. Rao, T. George, and K. Boaz, "Histochemical analysis of pathological alterations in oral lichen planus and oral lichenoid lesions.," Journal of oral science, vol. 48, no. 4, pp. 185-193, 2006.

[15] M.-J. Cobos-Fuentes, Á. Martínez-Sahuquillo-Márquez, I. Gallardo-Castillo, J.-R. Armas-Padrón, A. Moreno-Fernández, and P. Bullón-Fernández, "Oral lichenoid lesions related to contact with dental materials: a literature review," Medicina Oral, Patologia Oral y Cirugia Bucal, vol. 14, no. 10, pp. e514e520, 2009.

[16] S. G. Fitzpatrick, S. A. Hirsch, and S. C. Gordon, "The malignant transformation of oral lichen planus and oral lichenoid lesions: a systematic review," The Journal of the American Dental Association, vol. 145, no. 1, pp. 45-56, 2014.

[17] M. Ziemer, "Delayed-type cutaneous drug reactions. Pathogenesis, clinical features and histology," Der Hautarzt, vol. 65, no. 5, pp. 397-408, May 2014.

[18] G. W. Gynther, B. Rozell, and A. Heimdahl, "Direct oral microscopy and its value in diagnosing mucosal lesions a pilot study," Oral Surgery, Oral Medicine, Oral Pathology, Oral Radiology, and Endodontics, vol. 90, no. 2, pp. 164-170, 2000.

[19] D. J. Zegarelli, "Lichen planus: a simple and reliable biopsy technique," Journal of Oral Medicine, vol. 36, no. 1, pp. 18-20, 1981.

[20] L. S. Al-ani, "Oral lichen planus clinical study with the clinicopathological correlation in the diagnosis of O. L. P, Journal of College Dentistry, vol. 17, no. 1, pp. 57-60, 2005.

[21] M. Rad, M. A. Hashemipoor, A. Mojtahedi et al., "Correlation between clinical and histopathologic diagnoses of oral lichen planus based on modified WHO diagnostic criteria," Oral Surgery, Oral Medicine, Oral Pathology, Oral Radiology and Endodontology, vol. 107, no. 6, pp. 796-800, 2009.

[22] S. B. Ismail, S. K. S. Kumar, and R. B. Zain, "Oral lichen planus and lichenoid reactions: etiopathogenesis, diagnosis, management and malignant transformation," Journal of Oral Science, vol. 49, no. 2, pp. 89-106, 2007.

[23] E. H. van der Meij, K.-P. Schepman, and I. van der Waal, "The possible premalignant character of oral lichen planus and oral lichenoid lesions: a prospective study," Oral Surgery, Oral Medicine, Oral Pathology, Oral Radiology, and Endodontics, vol. 96, no. 2, pp. 164-171, 2003.

[24] E. H. van der Meij, H. Mast, and I. van der Waal, “The possible premalignant character of oral lichen planus and oral lichenoid lesions: A prospective five-year follow-up study of 192 patients," Oral Oncology, vol. 43, no. 8, pp. 742-748, 2007.

[25] G. D. Friedman, M. M. Asgari, E. M. Warton, J. Chan, and L. A. Habel, "Antihypertensive drugs and lip cancer in non-hispanic whites," Archives of Internal Medicine, vol. 172, no. 16, pp. 12461251, 2012.

[26] P. Arirachakaran, M. Hanvanich, P. Kuysakorn, and K. Thongprasom, "Antiretroviral drug-associated oral lichenoid reaction in HIV patient: a case report," International Journal of Dentistry, vol. 2010, Article ID 291072, 4 pages, 2010.

[27] P. Krogh, B. Hald, and P. Holmstrup, "Possible mycological etiology of oral mucosal cancer: catalytic potential of infecting Candida albicans and other yeasts in production of $\mathrm{N}$ nitrosobenzylmethylamine," Carcinogenesis, vol. 8, no. 10, pp. 1543-1548, 1987.

[28] G. Gannot, I. Gannot, H. Vered, A. Buchner, and Y. Keisari, "Increase in immune cell infiltration with progression of oral epithelium from hyperkeratosis to dysplasia and carcinoma," British Journal of Cancer, vol. 86, no. 9, pp. 1444-1448, 2002.

[29] E. Zancope, N. L. Costa, A. P. Junqueira-Kipnis et al., "Differential infiltration of $\mathrm{CD}^{+}$and NK cells in lip and oral cavity squamous cell carcinoma," Journal of Oral Pathology and Medicine, vol. 39, no. 2, pp. 162-167, 2010.

[30] W. J. Pichler and N. Yawalkar, "Allergic reactions to drugs: involvement of T cells," Thorax, vol. 55, no. 2, pp. S61-S65, 2000.

[31] M. J. Torres, C. Mayorga, and M. Blanca, "Nonimmediate allergic reactions induced by drugs: pathogenesis and diagnostic tests," Journal of Investigational Allergology and Clinical Immunology, vol. 19, no. 2, pp. 80-90, 2009. 


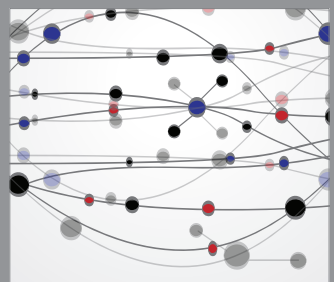

The Scientific World Journal
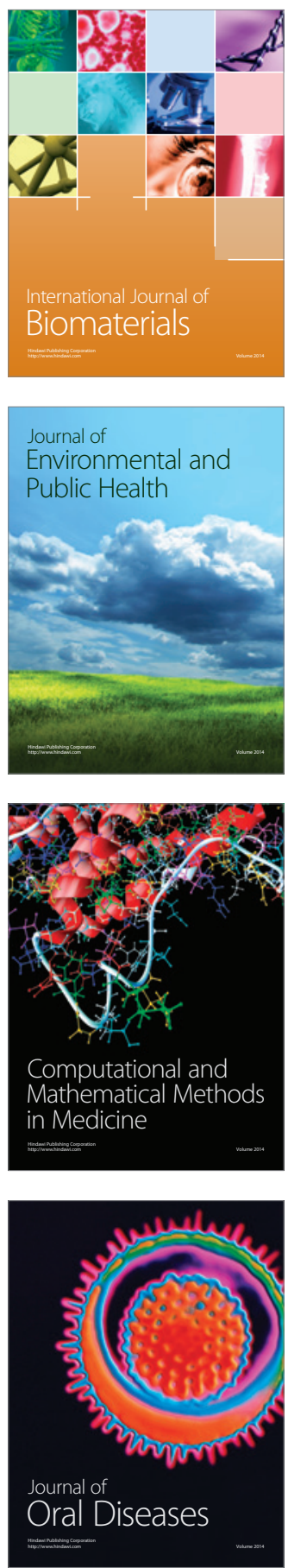
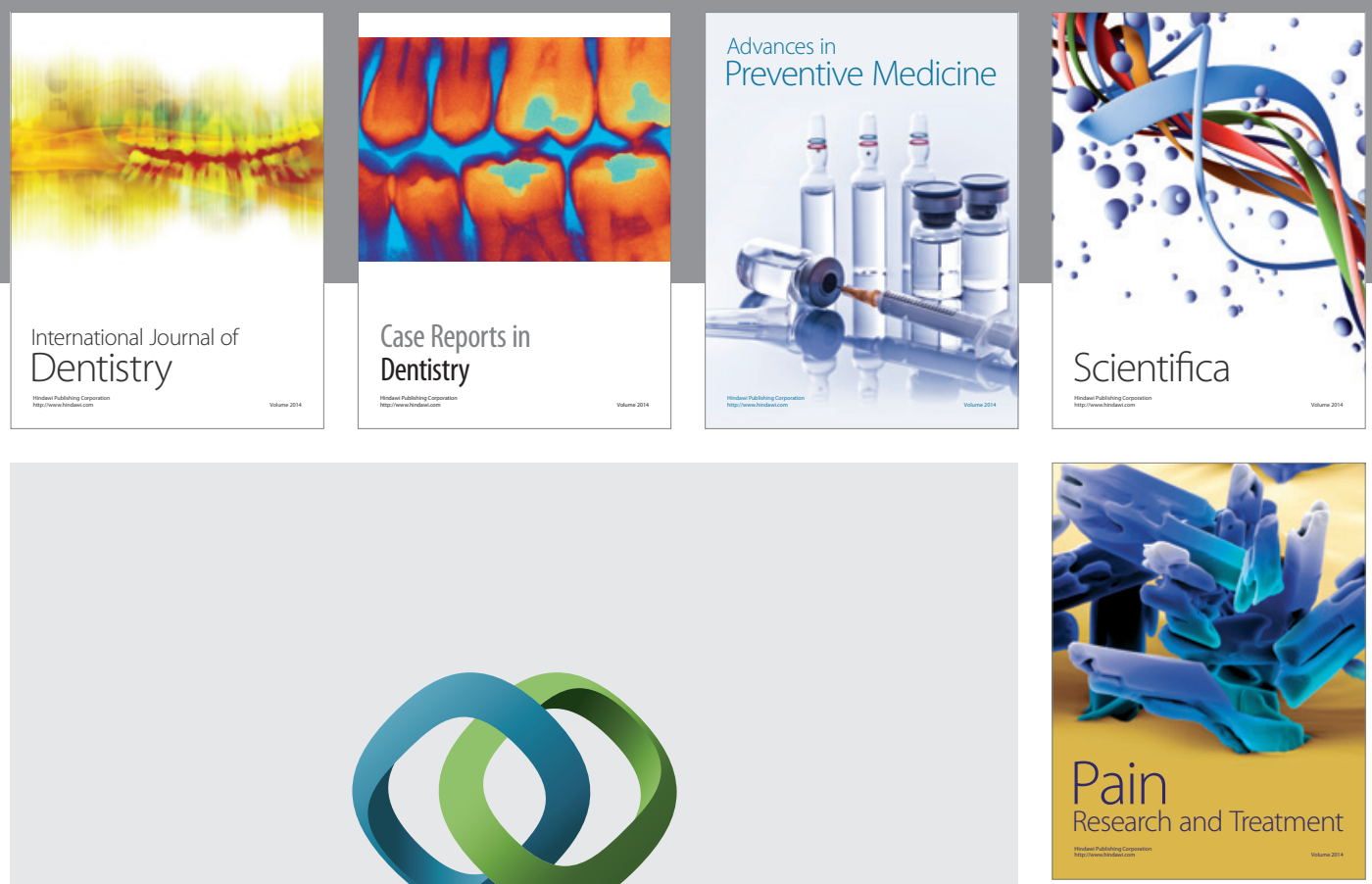

\section{Hindawi}

Submit your manuscripts at

http://www.hindawi.com
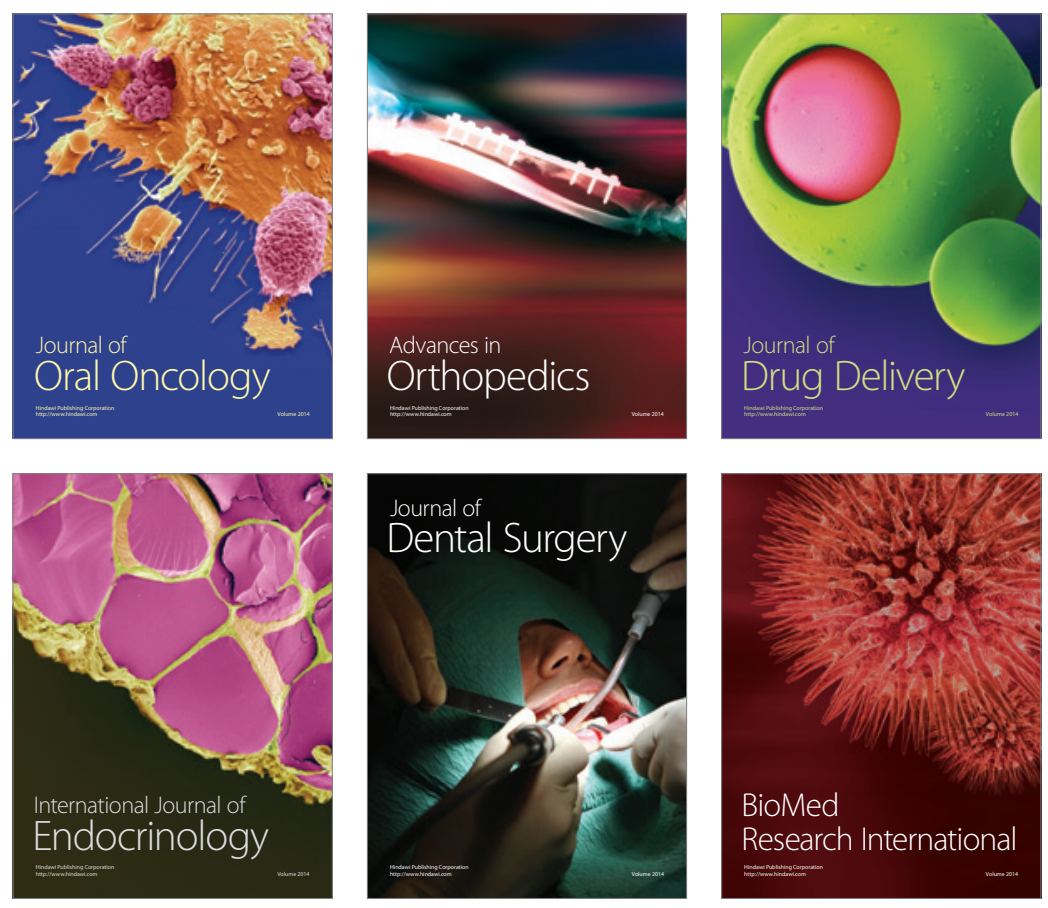

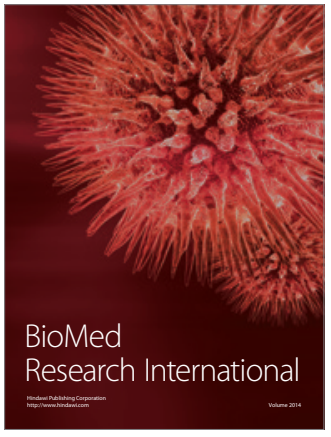

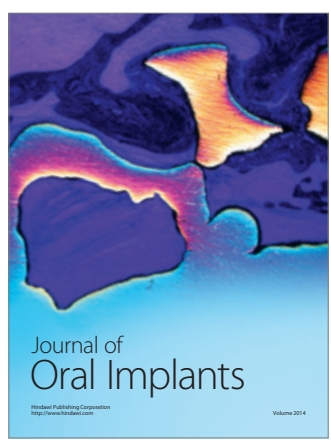
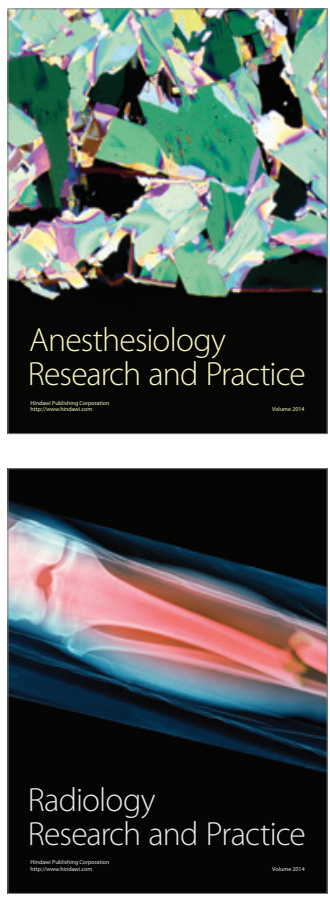